\title{
Series which are both max-plus and min-plus rational are unambiguous
}

\author{
Sylvain Lombardy and Jean Mairesse*
}

November 13, 2018

\begin{abstract}
Consider partial maps $\Sigma^{*} \longrightarrow \mathbb{R}$ with a rational domain. We show that two families of such series are actually the same: the unambiguous rational series on the one hand, and the max-plus and min-plus rational series on the other hand. The decidability of equality was known to hold in both families with different proofs, so the above unifies the picture. We give an effective procedure to build an unambiguous automaton from a max-plus automaton and a min-plus one that recognize the same series.
\end{abstract}

\section{Introduction}

A max-plus automaton is an automaton with multiplicities in the semiring $\mathbb{R}_{\max }=(\mathbb{R} \cup\{-\infty\}, \max ,+)$. Roughly, the transitions of the automaton have a label in a finite alphabet $\Sigma$ and a weight in the semiring. The weight of a word $w$ in $\Sigma^{*}$ is the maximum over all successful paths of label $w$ of the sum of the weights along the path. The series recognized by the automaton $\mathcal{T}$ is the resulting map $S(\mathcal{T}): \Sigma^{*} \rightarrow \mathbb{R}_{\max }$. The set of series recognized by a max-plus automaton is denoted by $\mathbb{R}_{\max } \operatorname{Rat}\left(\Sigma^{*}\right)$.

These automata, or the variants obtained by considering the min-plus semiring $\mathbb{R}_{\min }=(\mathbb{R} \cup\{+\infty\}$, min, +$)$ or subsemirings such as $\mathbb{Z}_{\max }$ or the tropical semiring $\mathbb{N}_{\text {min }}$, have been studied under various names: distance automata, cost automata, finance automata... The motivations range from complexity issues in formal language theory [15], to automatic speech recognition [11, via the modeling of Tetris heaps [5].

In Krob [10, the following question was raised: characterize the series which are recognized both by a max-plus and a min-plus automaton. That is, characterize the class $\mathbb{R}_{\max } \operatorname{Rat}\left(\Sigma^{*}\right) \cap \mathbb{R}_{\min } \operatorname{Rat}\left(\Sigma^{*}\right)$. Here, we answer the question by showing that these series are precisely the unambiguous max-plus (equivalently, minplus) series. Given a finitely ambiguous max-plus automaton, it is decidable if the corresponding series is unambiguous 8 . On the other hand, the status of

${ }^{*}$ LIAFA (UMR 7089), CNRS - Université Paris 7, 2 place Jussieu, 75251 Paris cedex 05, France, e-mail: \{lombardy, mairesse\}@liafa.jussieu.fr 
the same problem starting from an infinitely ambiguous max-plus automaton is unknown.

Apart from an interest in terms of classification, this result clarifies the status of the equality problem for max-plus series. The equality problem is to determine if " $S=T$ ", where $S$ and $T$ are series recognized by given max-plus automata. The equality problem is already undecidable in $\mathbb{Z}_{\max }$ and for two letters alphabet [9], but it is decidable for finitely ambiguous automata over $\mathbb{R}_{\max }$ [6, 17. Also, the following result is proved in [10]: if $\mathcal{A}$ is an automaton over $\mathbb{Z}_{\max }$, and $\mathcal{B}$ an automaton over $\mathbb{Z}_{\text {min }}$, then the problem " $S(\mathcal{A})=S(\mathcal{B})$ " is decidable, so the equality problem is decidable in $\mathbb{Z}_{\max } \operatorname{Rat}\left(\Sigma^{*}\right) \cap \mathbb{Z}_{\min } \operatorname{Rat}\left(\Sigma^{*}\right)$ (see Proposition 3.5). We can now conclude that the decidability result in [10] is a particular case of the one in [6, 17.

The paper is organized as follows. In $\$ 3$, we extend several results of [10] from $\mathbb{Z}_{\max }$ to $\mathbb{R}_{\max }$, in particular the so-called Fatou property. The results are then used in $\$ 4$ to obtain the characterization of $\mathbb{R}_{\max } \operatorname{Rat}\left(\Sigma^{*}\right) \cap \mathbb{R}_{\min } \operatorname{Rat}\left(\Sigma^{*}\right)$.

Below, the results on decidability and complexity should be interpreted under the assumption that two real numbers can be added or compared in constant time.

\section{Preliminaries}

Let $\mathbb{K}$ be any semiring and denote the neutral element of the additive, resp. multiplicative, law by $0_{\mathbb{K}}$, resp. $1_{\mathbb{k}}$. Let $Q$ be a finite set and $\Sigma$ a finite alphabet. A finite linear representation indexed by $Q$ over the alphabet $\Sigma$ and the semiring $\mathbb{K}$ is a triple $(\alpha, \mu, \beta)$, where $\alpha$, resp. $\beta$, is a row, resp. columm, vector of $\mathbb{K}^{Q}$ and $\mu$ is a morphism from $\Sigma^{*}$ into $\mathbb{K}^{Q \times Q}$ (for $u=u_{1} \cdots u_{n}, u_{i} \in \Sigma, \mu(u)=$ $\left.\mu\left(u_{1}\right) \cdots \mu\left(u_{n}\right)\right)$. The (formal power) series recognized by $(\alpha, \mu, \beta)$ is the series $S$ : $\Sigma^{*} \rightarrow \mathbb{K}$ such that $\langle S, w\rangle=\alpha \mu(w) \beta$. By the Schützenberger Theorem, the set of series that can be recognized by a finite linear representation is precisely the set of rational series. We denote it by $\mathbb{K R a t}\left(\Sigma^{*}\right)$.

Let $(\alpha, \mu, \beta)$ be a finite linear representation indexed by $Q$ over the semiring $\mathbb{K}$. This representation can be viewed as an automaton with set of states $Q$ : for every $(p, q)$ in $Q^{2}$ and every letter $a$ in $\Sigma$, if $\mu(a) \neq 0_{\mathbb{K}}$, there is a transition from $p$ to $q$ with label $a$ and weight $\mu(a)$. For every $p$ in $Q$, if $\alpha_{p} \neq 0_{\mathbb{K}}$, (resp. $\beta_{p} \neq 0_{\mathbb{K}}$ ), the state $p$ is initial with weight $\alpha_{p}$ (resp. terminal with weight $\beta_{p}$ ). In the sequel, we identify the linear representation with the corresponding automaton. As usual we transfer the terminology of graph theory to automata, e.g. (simple) path or circuit of an automaton. A path which is both starting with an ingoing arc and ending with an outgoing arc is called a successful path. The label of a path is the concatenation of the labels of the successive arcs (transitions). The weight of a path is the product (with respect to the multiplicative law of the semiring) of the weights of the successive arcs (including the ingoing and the outgoing arc, need it be). We denote by weight $(\pi)$ the weight of the path $\pi$. Two automata are equivalent if they recognize the same series. 
The support of a series $S$ is the set of words $w$ such that $\langle S, w\rangle \neq 0_{\mathbb{k}}$. We denote the support of $S$ by Supp $S$. The characteristic series of a language $L$ is the series $\mathbb{1}_{L}$ such that $\left\langle\mathbb{1}_{L}, w\right\rangle=1_{\mathbb{K}}$ if $w \in L$, and $\left\langle\mathbb{1}_{L}, w\right\rangle=0_{\mathbb{K}}$ otherwise.

The max-plus semiring $\mathbb{R}_{\max }$ is the semiring formed by the set $\mathbb{R} \cup\{-\infty\}$ with max as the additive operation and + as the multiplicative operation. In the sequel, we sometimes denote max and + respectively by $\oplus$ and $\otimes$; the neutral elements for these operations are respectively $-\infty$ and 0 . This semiring is naturally ordered by the usual order on $\mathbb{R}$ extended by: $\forall a,-\infty \leq a$. The minplus semiring $\mathbb{R}_{\min }$ is obtained by replacing max by min and $-\infty$ by $+\infty$ in the definition of $\mathbb{R}_{\max }$. The subsemirings $\mathbb{R}_{\max }^{-}, \mathbb{Z}_{\max }, \mathbb{Z}_{\text {max }}^{-}, \mathbb{Z}_{\min }, \ldots$, are defined in the natural way.

The subsemiring $\mathbb{B}=\{(-\infty, 0), \oplus, \otimes\}$ of $\mathbb{R}_{\max }$ is the Boolean semiring. There exists a morphism from $\mathbb{R}_{\max }$ onto $\mathbb{B}$ that maps $-\infty$ onto $-\infty$ and any other element onto 0 .

An automaton over $\mathbb{R}_{\max }$ is called a max-plus automaton, the corresponding series is called a max-plus (rational) series. Let $S$ be a max-plus rational series recognized by $(\alpha, \mu, \beta)$. Then Supp $S$ is the regular language recognized by the Boolean automaton obtained from $(\alpha, \mu, \beta)$ by applying to each coefficient the canonical morphism from $\mathbb{R}_{\max }$ onto $\mathbb{B}$.

An automaton is unambiguous if, for every word $w$, there is at most one successful path labeled by $w$. An automaton is 1-valued if, for every word $w$, all the successful paths labeled by $w$ have the same weight.

In $\mathbb{R}_{\max }$, a triple $(\alpha, \mu, \nu)$ is unambiguous if, for every word $w$,

a) there exists at most one $i,(\alpha \mu(w))_{i}+\beta_{i} \neq-\infty$

b) $\forall a \in \Sigma, \forall j$, there exists at most one $i,(\alpha \mu(w))_{i}+\mu(a)_{i j} \neq-\infty$.

In $\mathbb{R}_{\max }$, a triple $(\alpha, \mu, \nu)$ is 1-valued if, for every word $w$,

$$
\begin{aligned}
& \text { a) } \exists x_{w}, \forall i,(\alpha \mu(w))_{i}+\beta_{i} \in\left\{-\infty, x_{w}\right\} \\
& \text { b) } \forall a \in \Sigma, \forall j, \exists x, \forall i,(\alpha \mu(w))_{i}+\mu(a)_{i j} \in\{-\infty, x\} .
\end{aligned}
$$

Analogous definitions hold for triples over $\mathbb{R}_{\min }$. A max-plus, resp. min-plus, series is unambiguous if there exists an unambiguous max-plus, resp. min-plus, automaton recognizing it.

The operations on matrices over $\mathbb{R}_{\max }$ are defined classically with respect to the operations of $\mathbb{R}_{\max }$, e.g.: $(M \otimes N)_{i j}=\bigoplus_{k} M_{i k} \otimes M_{k j}=\max _{k}\left(M_{i k}+M_{k j}\right)$. We usually write $A B$ for $A \otimes B$. Given $u=\left(u_{1}, \ldots, u_{n}\right) \in \mathbb{R}_{\max }^{n}$ and $\lambda \in \mathbb{R}_{\max }$, set $\lambda u=\left(\lambda \otimes u_{1}, \ldots, \lambda \otimes u_{n}\right)=\left(\lambda+u_{1}, \ldots, \lambda+u_{n}\right)$.

Consider a matrix $A \in \mathbb{R}_{\max }^{Q \times Q}$. The matrix $A$ is irreducible if the graph of $A$ (nodes $Q, i \rightarrow j$ if $A_{i j} \neq-\infty$ ) is strongly connected. A scalar $\lambda \in \mathbb{R}_{\max }$ and a column vector $u \in \mathbb{R}_{\max }^{Q} \backslash(-\infty, \ldots,-\infty)$ such that

$$
A u=\lambda u=\left(\lambda+u_{i}\right)_{i \in Q},
$$


are called respectively an eigenvalue and an eigenvector of $A$. The number of eigenvalues is at least one and at most $|Q|$, and it is exactly one if $A$ is irreducible. The max-plus spectral theory is the study of these eigenvalues and eigenvectors. In the sequel, we only need the result in Theorem 2.1. For a more complete picture, as well as proofs and bibliographic references, see for instance [1.

Theorem 2.1 (Max-plus spectral theory). Consider $A \in \mathbb{R}_{\max }^{Q \times Q}$. Let $\rho(A)$ be the maximal eigenvalue of $A$. We have:

$$
\rho(A)=\max _{k \leq|Q|} \max _{i_{1}, \ldots, i_{k-1} \in Q} \frac{A_{i_{1} i_{2}}+A_{i_{2} i_{3}}+\cdots A_{i_{k-1} i_{1}}}{k}=\max _{k \leq|Q|} \max _{i \in Q} \frac{A_{i i}^{k}}{k} .
$$

In words, $\rho(A)$ is the maximal mean weight of a simple circuit of (the graph of) A.

\section{Some decidability results}

In this section, we reconsider the various results proved by Krob [10] for series in $\mathbb{Z}_{\max }$ and we extend them to $\mathbb{R}_{\max }$. The proofs are different since they use the max-plus spectral theory. The results are then used in 4 Obviously, analogous results hold for $\mathbb{R}_{\min }$.

The decidability part of Proposition 3.1 is given in [10, Corollary 4.3] for series in $\mathbb{Z}_{\max } \operatorname{Rat}\left(\Sigma^{*}\right)$. The proof in [10] is different and relies on the fact that $\mathbb{Z}_{\max } \operatorname{Rat}\left(\Sigma^{*}\right)$ is a constructive Fatou extension of $\mathbb{Z}_{\max }^{-} \operatorname{Rat}\left(\Sigma^{*}\right)$. We prove a generalization of this last result for $\mathbb{R}_{\max } \operatorname{Rat}\left(\Sigma^{*}\right)$ in Proposition 3.2 below. Using Proposition 3.2, we can then recover the decidability in Proposition 3.1 in the same way as in [10]. Observe however that the proof of Proposition 3.1 given below provides a polynomial procedure.

In contrast with Proposition 3.1 the problem " $\forall w \in \Sigma^{*},\langle S, w\rangle \geq 0$ " is undecidable even for $S \in \mathbb{Z}_{\max } \operatorname{Rat}\left(\Sigma^{*}\right)$, see $[9$.

Proposition 3.1. Consider the following problem:

$$
\begin{array}{ll}
\text { Instance: } & S \in \mathbb{R}_{\max } \operatorname{Rat}\left(\Sigma^{*}\right) \\
\text { Problem: } & \forall w \in \Sigma^{*},\langle S, w\rangle \leq 0 .
\end{array}
$$

This problem can be decided with an algorithm of polynomial time complexity in the size of an automaton recognizing $S$.

Proof. Let $\mathcal{A}=(\alpha, \mu, \beta)$ be a trim automaton recognizing $S$ with set of states Q. Set

$$
M=\bigoplus_{a \in \Sigma} \mu(a)
$$

Let $\rho(M)$ be the maximal eigenvalue of $M$. By the Max-plus Spectral Theorem 2.1. there exist $k \in \mathbb{N}^{*}$ and $i \in Q$ such that $M_{i i}^{k}=k \times \rho(M)$. It implies 
that there exists $w \in \Sigma^{k}$ such that $\mu(w)_{i i}=k \times \rho(M)$. Clearly, we have $\mu\left(w^{n}\right)_{i i} \geq n \times k \times \rho(M)$ for all $n \in \mathbb{N}^{*}$. Since the automaton is trim, there exist $w_{1}, w_{2} \in \Sigma^{*}$ such that $\alpha \mu\left(w_{1}\right)_{i}>-\infty$ and $\mu\left(w_{2}\right) \beta_{i}>-\infty$. Assume that $\rho(M)>0$, then by choosing $n$ large enough, we get the following contradiction

$$
\left\langle S, w_{1} w^{n} w_{2}\right\rangle \geq \alpha \mu\left(w_{1}\right)_{i}+\mu\left(w^{n}\right)_{i i}+\mu\left(w_{2}\right) \beta_{i}>0 .
$$

Assume now that $\rho(M) \leq 0$. By the Max-plus Spectral Theorem 2.1 it implies that all the circuits in the automaton have a weight which is non-positive. Assume that there exists a word $w$ such that $\langle S, w\rangle>0$. Let $\pi$ be a successful path of label $w$ and maximal weight in the automaton. If $\pi$ contains a circuit, then the path $\pi^{\prime}$ obtained by removing the circuit is still a successful path. In particular, if $w^{\prime}$ is the label of $\pi^{\prime}$, we have $\left\langle S, w^{\prime}\right\rangle \geq\langle S, w\rangle>0$. So we can choose, without loss of generality, a word $w$ such that $\langle S, w\rangle>0$ and $|w|<|Q|$. Now notice that we have for all $k \in \mathbb{N}$,

$$
\left(\exists u \in \Sigma^{k},\langle S, u\rangle>0\right) \Longleftrightarrow \alpha M^{k} \beta>0 .
$$

Summarizing the results obtained so far, we get

$$
\left(\forall u \in \Sigma^{*},\langle S, u\rangle \leq 0\right) \Longleftrightarrow(\rho(M) \leq 0) \wedge\left(\forall k \in\{0, \ldots,|Q|-1\}, \alpha M^{k} \beta \leq 0\right),
$$

where $M^{0}$ is the identity matrix defined by: $\forall i, M_{i i}^{0}=0, \forall i \neq j, M_{i j}^{0}=-\infty$.

Complexity. Computing the matrix $M$ has a time complexity $O\left(|\Sigma||Q|^{2}\right)$. Computing $\rho(M)$ can be done using Karp algorithm [1, Theorem 2.19] in time $O\left(|Q|^{3}\right)$. Computing $\alpha M^{k} \beta$ for all $k \in\{0, \ldots,|Q|-1\}$ requires also a time complexity $O\left(|Q|^{3}\right)$.

Proposition 3.2 is proved for series in $\mathbb{Z}_{\max } \operatorname{Rat}\left(\Sigma^{*}\right)$ in [10, Proposition 4.2]. It is not obvious to extend the approach of [10] to series in $\mathbb{R}_{\max } \operatorname{Rat}\left(\Sigma^{*}\right)$. We propose a quite different proof.

Proposition 3.2 (Fatou property). Consider a series $S$ in $\mathbb{R}_{\max } \operatorname{Rat}\left(\Sigma^{*}\right)$. Then we have

$$
S: \Sigma^{*} \longrightarrow \mathbb{R}_{\max }^{-} \Longrightarrow S \in \mathbb{R}_{\max }^{-} \operatorname{Rat}\left(\Sigma^{*}\right) .
$$

Furthermore, given an automaton $\mathcal{A}$ over $\mathbb{R}_{\max }$ recognizing $S$, one can effectively compute an automaton $\mathcal{A}^{-}$over $\mathbb{R}_{\max }^{-}$recognizing $S$. The procedure to get $\mathcal{A}^{-}$ from $\mathcal{A}$ has a polynomial time complexity in the size of $\mathcal{A}$.

Proof. Let $(\alpha, \mu, \beta)$ be a trim triple recognizing $S$ with set of states $\{1, \ldots, n\}$. Define the matrix $M=\bigoplus_{a \in \Sigma} \mu(a)$. Since $S: \Sigma^{*} \longrightarrow \mathbb{R}_{\max }^{-}$, it follows from (1) that $\rho(M) \leq 0$. In particular any circuit has non-positive weight. It follows immediately that:

$$
M^{*}=\bigoplus_{i \in \mathbb{N}} M^{i}=I \oplus M \oplus M^{2} \oplus \cdots \oplus M^{n-1},
$$


where $I$ is the identity matrix of dimension $n \times n$ defined by $\forall i, I_{i i}=0, \forall i \neq$ $j, I_{i j}=-\infty$. Since $S: \Sigma^{*} \longrightarrow \mathbb{R}_{\max }^{-}$, it follows that $\alpha M^{*} \beta \leq 0$. Set $u=M^{*} \beta$ and define the diagonal matrix (the non-diagonal coefficients being $-\infty$ ) $D=$ $\operatorname{diag}\left(u_{1}, \ldots, u_{n}\right)$. Define

$$
\widehat{\alpha}=\alpha D, \quad \widehat{\beta}=D^{-1} \beta, \quad \forall a \in \Sigma, \widehat{\mu}(a)=D^{-1} \mu(a) D .
$$

Clearly, the automaton $(\widehat{\alpha}, \widehat{\mu}, \widehat{\beta})$ recognizes the series $S$. We have: $\forall i, \widehat{\alpha}_{i}=$ $\alpha_{i}+\left(M^{*} \beta\right)_{i} \leq \alpha M^{*} \beta \leq 0$; and also: $\forall i, \widehat{\beta}_{i}=\beta_{i}-\left(M^{*} \beta\right)_{i} \leq \beta_{i}-\beta_{i}=0$. At last, we have: $\forall a \in \Sigma, \forall i$,

$$
\begin{aligned}
\bigoplus_{j} \widehat{\mu}(a)_{i j} \leq \bigoplus_{j}\left(D^{-1} M D\right)_{i j} & =\bigoplus_{j}\left[\left(D^{-1} M\right)_{i j}+\left(M^{*} \beta\right)_{j}\right] \\
& =\left(D^{-1} M M^{*} \beta\right)_{i} \leq\left(D^{-1} M^{*} \beta\right)_{i}=0,
\end{aligned}
$$

where we have used that $M M^{*} \leq I \oplus M M^{*}=M^{*}$. Hence the triple $(\widehat{\alpha}, \widehat{\mu}, \widehat{\beta})$ is defined over the semiring $\mathbb{R}_{\max }^{-}$. This completes the proof.

Complexity. The matrix $M$ is computed in time $O\left(|\Sigma| n^{2}\right)$. Then, computing $u=M^{*} \beta$ requires $O\left(n^{3}\right)$ operations. Knowing $u$, computing $(\widehat{\alpha}, \widehat{\mu}, \widehat{\beta})$ requires $O\left(|\Sigma| n^{3}\right)$ operations.

Proposition 3.3 is proved for series in $\mathbb{Z}_{\max } \operatorname{Rat}\left(\Sigma^{*}\right)$ in [10, Proposition 5.1]. The proof relies on the Fatou property. Since we have extended this last property to $\mathbb{R}_{\max } \operatorname{Rat}\left(\Sigma^{*}\right)$, the proof of Krob carries over unchanged. In the proof below, we present the arguments in a slightly different way.

Proposition 3.3. The following problem is decidable:

$$
\begin{array}{ll}
\text { Instance: } & S \in \mathbb{R}_{\max } \operatorname{Rat}\left(\Sigma^{*}\right) \text { and } c \in \mathbb{R} \\
\text { Problem: } & \forall w \in \Sigma^{*},\langle S, w\rangle=c \quad(\text { i.e. } S=c) .
\end{array}
$$

Proof. First of all, it is enough to prove the result for $c=0$. Indeed, testing if $\langle S, w\rangle=c$ is equivalent to testing if $\left\langle S^{\prime}, w\right\rangle=0$ where $S^{\prime}$ is the series defined by $\left\langle S^{\prime}, u\right\rangle=\langle S, u\rangle-c$. And it is straightforward to get a triple recognizing $S^{\prime}$ from a triple recognizing $S$.

According to Proposition 3.1 we can decide if $S$ belongs to $\mathbb{R}_{\max }^{-}\left(\Sigma^{*}\right)$. If not, then we have $S \neq 0$. If $S \in \mathbb{R}_{\max }^{-}\left(\Sigma^{*}\right)$ then, by Proposition 3.2 there exists an effectively computable automaton $(\alpha, \mu, \beta)$ over $\mathbb{R}_{\max }^{-}$recognizing $S$. We define an automaton $(\bar{\alpha}, \bar{\mu}, \bar{\beta})$ as follows:

$$
\forall a \in \Sigma, \forall i, j, \bar{\mu}(a)_{i j}=\left\{\begin{array}{ll}
0 & \text { if } \mu(a)_{i j}=0 \\
-\infty & \text { if } \mu(a)_{i j}<0
\end{array},\right.
$$

with $\bar{\alpha}$ and $\bar{\beta}$ being defined from $\alpha$ and $\beta$ in the same way. The important property is that for $w \in \Sigma^{*}$,

$$
\langle S, w\rangle=0 \Longleftrightarrow \bar{\alpha} \bar{\mu}(w) \bar{\beta}=0 .
$$


Let us set $\bar{\mu}\left(\Sigma^{*}\right)=\left\{\bar{\mu}(w), w \in \Sigma^{*}\right\}$. Obviously, $\left(\bar{\mu}\left(\Sigma^{*}\right), \otimes\right)$ is a submonoid of the finite monoid $\left(\mathbb{B}^{n \times n}, \otimes\right)$. In particular, $\bar{\mu}\left(\Sigma^{*}\right)$ is finite and can be effectively constructed. In view of (2), we have

$$
\left(\forall w \in \Sigma^{*},\langle S, w\rangle=0\right) \Longleftrightarrow\left(\forall A \in \bar{\mu}\left(\Sigma^{*}\right), \bar{\alpha} A \bar{\beta}=0\right) .
$$

Since $\bar{\mu}\left(\Sigma^{*}\right)$ is finite and effectively computable, the property on the right can be checked algorithmically.

Complexity. In contrast with Proposition 3.1, we do not get a polynomial procedure in Proposition 3.3. Deciding if the right-hand side in (3) holds is PSPACE-complete with respect to the dimension of the triple, see for instance 7. Theorem 13.14 and Exercise 13.25]. This is known as the universality problem.

Proposition 3.4. The following problem is decidable:

$$
\begin{array}{ll}
\text { Instance: } & S \in \mathbb{R}_{\max } \operatorname{Rat}\left(\Sigma^{*}\right) \text { and } c \in \mathbb{R} \\
\text { Problem: } & \forall w \in \operatorname{Supp} S,\langle S, w\rangle=c .
\end{array}
$$

Proof. The proof is the same as in Proposition 3.3. Instead of deciding the right-hand side of (3), it must be decided whether $(\alpha, \mu, \beta)$ and $(\bar{\alpha}, \bar{\mu}, \bar{\beta})$ have the same support.

Complexity. The complexity of this problem is PSPACE-complete. Indeed, $(\bar{\alpha}, \bar{\mu}, \bar{\beta})$ is obtained from $(\alpha, \mu, \beta)$ by deleting some transitions. And deciding whether the language accepted by a non-deterministic automaton remains the same after the deletion of some transitions is PSPACE-complete. We briefly explain why. First, the equivalence problem for non-deterministic Boolean automata is PSPACE-complete [16, and thus our problem is in PSPACE. Next, let $\mathcal{A}$ be a non-deterministic automaton and let $\mathcal{A}^{\prime}$ be the automaton obtained from $\mathcal{A}$ by adding a state, initial and terminal, with loops labelled by evey letter. Deciding whether $\mathcal{A}^{\prime}$ is equivalent to $\mathcal{A}$ is equivalent to deciding whether $\mathcal{A}$ accepts every word (universality problem), which is PSPACE-complete. Thus our problem is PSPACE-hard.

Proposition 3.5 is proved for series in $\mathbb{Z}_{\max } \operatorname{Rat}\left(\Sigma^{*}\right)$ and $\mathbb{Z}_{\min } \operatorname{Rat}\left(\Sigma^{*}\right)$ in 10, Proposition 5.3]. As discussed in the Introduction, a consequence of Proposition 3.5 is that the equality problem is decidable in $\mathbb{R}_{\max } \operatorname{Rat}\left(\Sigma^{*}\right) \cap \mathbb{R}_{\min } \operatorname{Rat}\left(\Sigma^{*}\right)$. Quoting [10]: "the problem remains to characterize (such) series". This is done in 4

Proposition 3.5. The following problem is decidable:

$$
\begin{array}{ll}
\text { Instance: } & S \in \mathbb{R}_{\max } \operatorname{Rat}\left(\Sigma^{*}\right), T \in \mathbb{R}_{\min } \operatorname{Rat}\left(\Sigma^{*}\right) \\
\text { Problem: } & S=T .
\end{array}
$$

The above equality should be interpreted as: Supp $S=\operatorname{Supp} T$ and $\forall w \in$ Supp $S,\langle S, w\rangle=\langle T, w\rangle$. 
Proof. Define the series $-T$ with coefficients in $\mathbb{R} \cup\{+\infty\}$ by $\langle-T, w\rangle=-\langle T, w\rangle$ for all $w$. Clearly $-T \in \mathbb{R}_{\max } \operatorname{Rat}\left(\Sigma^{*}\right)$. The above problem is equivalent to:

$$
\text { (a) Supp } S=\operatorname{Supp} T \text { and }(b) \forall w \in \operatorname{Supp} S,\langle S-T, w\rangle=0 \text {. }
$$

Point (a) is the problem of equivalence of rational languages and is thus decidable. The series $S-T$ is the Hadamard max-plus product of $S$ and $-T$; it is recognized by the tensor product of triples recognizing $S$ and $-T$ :

Let $(\alpha, \mu, \nu)$ (resp. $\left.\left(\alpha^{\prime}, \mu^{\prime}, \nu^{\prime}\right)\right)$ be a trim triple recognizing $S$ (resp. $-T$ ) with set of states $Q=\{1, \ldots, n\}$ (resp. $Q^{\prime}=\{1, \ldots, m\}$ ). Let $(\iota, \pi, \tau)$ be the triple defined on $Q \times Q^{\prime}$ by:

$$
\iota_{p, q}=\alpha_{p}+\alpha_{q}^{\prime} \quad \tau_{p, q}=\nu_{p}+\nu_{q}^{\prime} \quad \pi(a)_{(p, q)(r, s)}=\mu(a)_{p r}+\mu^{\prime}(a)_{q s} .
$$

By Proposition 3.4, (b) is decidable.

Using the same proof, one also shows that " $S \leq T$ " is decidable. On the other hand, " $S \geq T$ " is already undecidable for $S \in \mathbb{Z}_{\max } \operatorname{Rat}\left(\Sigma^{*}\right)$ and $T \equiv 0$, see [9].

\section{Max-plus and min-plus rational implies un- ambiguous}

To prove that a series recognized by a max-plus and a min-plus automaton is also recognized by an unambiguous automaton, we use an intermediate step which is to prove that it is recognized by a 1-valued automaton.

Recall that the notion of 1-valuedness of a max-plus automaton has been defined in 92 This notion clearly extends to any automaton with multiplicities over an idempotent semiring, in particular to a transducer. A transducer $\mathcal{T}$ is an automaton over the semiring $\operatorname{BRat}\left(B^{*}\right)$. The transducer $\mathcal{T}$ is 1-valued (or functional) if $|\operatorname{Supp}\langle S(\mathcal{T}), w\rangle| \leq 1$ for all $w$. Next result is classical and due to Eilenberg [4] and Schützenberger [14, see [2, Chapter IV.4]: a 1-valued transducer can be effectively transformed into an equivalent unambiguous one. The proof of Eilenberg and Schützenberger easily extends to a 1-valued automaton with multiplicities in an idempotent semiring. Here we give a different and simple proof of the same result. The argument is basically the same one as in 8 , Section 4].

Proposition 4.1. For any max-plus or min-plus 1-valued automaton, there exists an unambiguous automaton which recognizes the same series.

Proof. Let $\mathcal{A}=(\alpha, \mu, \nu)$ be a 1-valued automaton and $\mathcal{A}^{\prime}$ the underlying Boolean automaton. Let $\mathcal{D}=(\beta, \delta, \gamma)$ be the determinized automaton of $\mathcal{A}^{\prime}$ obtained by the subset construction. Let $\mathcal{S}=(\iota, \pi, \tau)$ be the tensor product of $\mathcal{A}$ and $\mathcal{D}$ :

$$
\iota_{p, q}=\alpha_{p}+\beta_{q}, \quad \tau_{p, q}=\nu_{p}+\gamma_{q}, \quad \pi(a)_{(p, q)(r, s)}=\mu(a)_{p r}+\delta(a)_{q s} .
$$


The automaton $\mathcal{S}$ is the Schützenberger covering of $\mathcal{A}$, see [13]. There is a competition in $\mathcal{S}$ if:

(a) there exist $q, r, s, p$, and $p^{\prime}$ such that $p \neq p^{\prime}, \pi(a)_{(p, q)(r, s)} \neq-\infty$ and $\pi(a)_{\left(p^{\prime}, q\right)(r, s)} \neq-\infty$, or

(b) there exist $q, p$ and $p^{\prime}$ such that $p \neq p^{\prime}, \tau_{p, q} \neq-\infty$ and $\tau_{p^{\prime}, q} \neq-\infty$.

Let $\mathcal{U}$ be any automaton obtained from $\mathcal{S}$ by removing the minimal number of transitions and/or terminal arrows such that there is no more competition. We claim that $\mathcal{U}$ is an unambiguous automaton equivalent to $\mathcal{A}$. The proof of this claim can be found in [8, Section 4].

As a side remark, the above proof is also clearly valid in any idempotent semiring.

We now have all the ingredients to prove the main result.

Proposition 4.2. Let $S$ be a series in $\mathbb{R}_{\max } \operatorname{Rat}\left(\Sigma^{*}\right)$. The series $-S$ is in $\mathbb{R}_{\max } \operatorname{Rat}\left(\Sigma^{*}\right)$ if and only if the series $S$ is unambiguous.

Proof. Let $\mathcal{A}=(\alpha, \mu, \nu)$, resp. $\mathcal{A}^{\prime}=\left(\alpha^{\prime}, \mu^{\prime}, \nu^{\prime}\right)$, be a triple that recognizes $S$, resp. $-S$. Let $\mathcal{P}=(\iota, \pi, \tau)$ be the triple on the semiring $\mathbb{R}_{\max } \times \mathbb{R}_{\max }$ and with set of states $Q \times Q^{\prime}$ defined by:

$$
\begin{gathered}
\iota_{p, q}=\left(\alpha_{p}, \alpha_{p}+\alpha_{q}^{\prime}\right), \quad \tau_{p, q}=\left(\nu_{p}, \nu_{p}+\nu_{q}^{\prime}\right) \\
\pi(a)_{(p, q)(r, s)}=\left(\mu(a)_{p r}, \mu(a)_{p r}+\mu^{\prime}(a)_{q s}\right)
\end{gathered}
$$

This triple recognizes the series $(S, S-S)=\left(S, \mathbb{1}_{\text {Supp } S}\right)$.

For every vector or matrix $x$ with coefficients in $\mathbb{R}_{\max }^{2}$, for $i$ in $\{1,2\}$, we denote $x^{(i)}$, the projection of $x$ with respect to the $i$-th coordinate.

By Proposition 3.2 there exists an automaton $\left(\iota^{\prime}, \pi^{\prime}, \tau^{\prime}\right)$ equivalent to $(\iota, \pi, \tau)$ and such that $\left(\iota^{\prime(2)}, \pi^{\prime(2)}, \tau^{\prime(2)}\right)$ is over $\mathbb{R}_{\max }^{-}$(the first ccordinate is unmodified: $\left.\iota^{\prime(1)}=\iota^{(1)}, \pi^{\prime(1)}=\pi^{(1)}, \tau^{\prime(1)}=\tau^{(1)}\right)$. We define an automaton $\mathcal{B}=(\bar{\iota}, \bar{\pi}, \bar{\tau})$ over the semiring $\mathbb{R}_{\max }$ as follows:

$$
\forall a \in \Sigma, \forall i, j \in Q \times Q^{\prime}, \bar{\pi}(a)_{i j}=\left\{\begin{array}{ll}
\pi^{(1)}(a)_{i j} & \text { if } \pi^{\prime(2)}(a)_{i j}=0 \\
-\infty & \text { if } \pi^{\prime(2)}(a)_{i j}<0
\end{array},\right.
$$

with $\bar{\iota}$ and $\bar{\tau}$ being defined from $\iota^{\prime}$ and $\tau^{\prime}$ in the same way. We claim that $(\bar{\imath}, \bar{\pi}, \bar{\tau})$ is a 1 -valued automaton that recognizes $S$.

For every word $w$, every successful path of $\mathcal{B}$ labeled by $w$ has a weight equal to the first coordinate $k_{1}$ of the weight $k$ of a successful path of $\mathcal{P}$ such that $k_{2}=0$. It means that $k_{1}$ is the weight of a successful path labeled by $w$ in $\mathcal{A}$ and that $-k_{1}$ is the weight of a successful path labeled by $w$ in $\mathcal{A}^{\prime}$. Hence, $k_{1} \leq\langle S, w\rangle$ and $-k_{1} \leq\langle-S, w\rangle$, and so $k_{1}=\langle S, w\rangle$. Therefore, every successful path of $\mathcal{B}$ labeled by $w$ has a weight equal to $\langle S, w\rangle$.

Conversely, every word $w$ in Supp $S$ labels a successful path in $\mathcal{B}$. Indeed, there is a successful path labeled by $w$ with weight $\langle S, w\rangle$ in $\mathcal{A}$, and a successful path labeled by $w$ with weight $-\langle S, w\rangle$ in $\mathcal{A}^{\prime}$. The product of the two paths gives 
a successful path in $\mathcal{P}$ labeled by $w$ with a weight having a second coordinate equal to 0 , hence, after applying Proposition 3.2 the weight of every transition along this path has a second coordinate equal to 0 .

Therefore $\mathcal{B}$ recognizes the same series as $\mathcal{A}$. We complete the proof by applying Proposition 4.1 .

There is a canonical bijection from $\mathbb{R}_{\max }$ to $\mathbb{R}_{\min }$ that consists in mapping every $x$ different from $-\infty$ onto itself and $-\infty$ onto $+\infty$. This bijection is obviously not an isomorphism. With some abuse, we say that a series $S$ of $\mathbb{R}_{\max } \operatorname{Rat}\left(\Sigma^{*}\right)$ is also in $\mathbb{R}_{\min } \operatorname{Rat}\left(\Sigma^{*}\right)$ if its image with respect to the canonical bijection is in $\mathbb{R}_{\min } \operatorname{Rat}\left(\Sigma^{*}\right)$.

Corollary 4.3. A series $S$ is in $\mathbb{R}_{\max } \operatorname{Rat}\left(\Sigma^{*}\right) \cap \mathbb{R}_{\min } \operatorname{Rat}\left(\Sigma^{*}\right)$ if and only if it is unambiguous. Starting from a pair formed by a max-plus and a min-plus automaton recognizing $S$, one can effectively compute an unambiguous automaton recognizing $S$.

Observe that given a pair formed by a max-plus and a min-plus automaton, it can be checked if they indeed recognize the same series using Proposition 3.5.

Proof. Since $S$ is in $\mathbb{R}_{\min } \operatorname{Rat}\left(\Sigma^{*}\right),-S$ is in $\mathbb{R}_{\max } \operatorname{Rat}\left(\Sigma^{*}\right)$ (there is an isomorphism from $\mathbb{R}_{\max }$ onto $\mathbb{R}_{\min }$ that maps $x$ onto $\left.-x\right)$. This result is therefore equivalent to Proposition 4.2. The effective computation of an unambiguous automaton recognizing $S$ is done in the proof of Proposition 4.2

Complexity. In Corollary 4.3, one gets a 1-valued automaton recognizing $S$ of dimension the product of the dimensions of the max-plus and min-plus automata. This follows directly from the proof of Proposition 4.2. The time complexity to construct it is also clearly polynomial. On the other hand, the dimension of an unambiguous automaton recognizing $S$ may be exponential with respect to the dimension of the 1 -valued automaton.

\section{$5 \quad$ Examples}

Let $S$ be the series defined by $\langle S, w\rangle=\max \left(|w|_{a},|w|_{b}\right)$. This series is obviously max-plus rational. In [8, it is proved that $S$ is not unambiguous (section 3.2), and with a different argument that it is not min-plus rational (section 3.6). We know now that both statements are equivalent.

We consider now a simple example on which we illustrate the different steps of our proof.

Let $\mathcal{A}_{\max }$ and $\mathcal{B}_{\min }$ be the two automata drawn on Figure 1-1a) (the weights equal to 0 on ingoing or outgoing arrows have been omitted). The automaton $\mathcal{A}_{\text {max }}$ is a max-plus automaton, while the automaton $\mathcal{B}_{\text {min }}$ is a min-plus automaton. Their product, performed as in the proof of Proposition 4.2, is drawn on the same figure. The automata are equivalent only if the weight with respect to the 

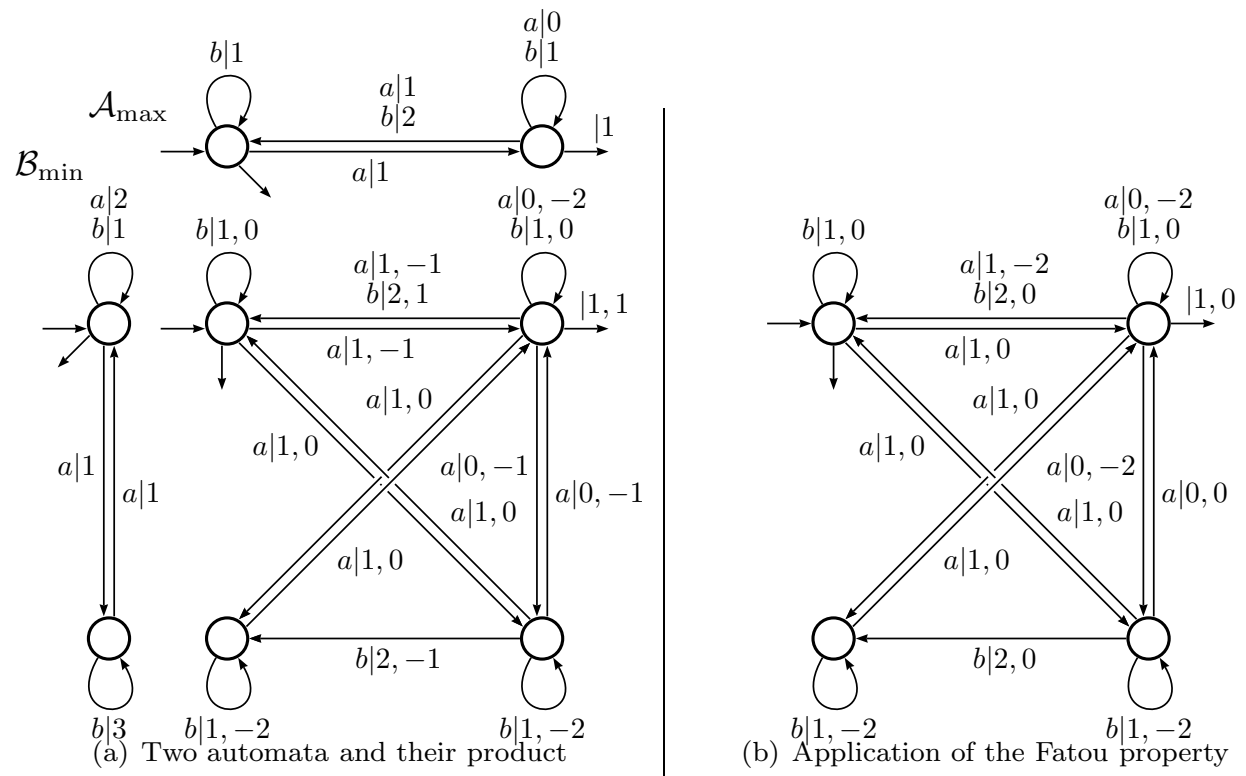

Figure 1: Getting an unambiguous automaton (I)

second coordinate is non-positive on every successful path. Hence, we can apply the Fatou property (Proposition 3.2) to get an equivalent automaton on which the weight ot the second coordinate is non-positive on every arc (transitions, and initial and final arrows). The result is shown on Figure1f(b). After deleting the arcs that have a second coordinate weight different from 0 , and remembering only the first coordinate, we get the 1-valued automaton of Figure 2-(a). As this automaton has the same support as $\mathcal{A}_{\max }$ and $\mathcal{B}_{\min }$, we can conclude that $\mathcal{A}_{\max }$ and $\mathcal{B}_{\text {min }}$ are indeed equivalent. We can then turn this 1-valued automaton into an unambiguous one (Figure 2-(b)), using the construction of Proposition 4.1. This example is "artificial". For instance, we can get an equivalent two states unambiguous automaton from the max-plus one only by deleting some transitions. This does not imply that there always exists an unambiguous automaton that has a number of states less or equal to the number of states of either the max-plus or the min-plus automaton. We now give an example that enhances this point.

Recall first that every max-plus or min-plus series over a one-letter alphabet is unambiguous [3, 12. We now make the following claim (the proof is not difficult): If $S$ is a max-plus rational series over the one-letter alphabet $\{a\}$, and if the sequence $\left(\left\langle S, a^{n}\right\rangle\right)_{n \in \mathbb{N}}$ is periodic of minimal period $p$, then the smallest 1 -valued automaton recognizing $S$ is of dimension $p$, and is deterministic.

Let $p, q, r$, and $s$ be four distinct prime numbers. For $i \in\{p, q, r, s\}$, define the series $S_{i}$ on $\{a\}^{*}$ by:

$$
\text { Supp } S_{i}=\left\{a^{n} \mid n=0 \quad \bmod i\right\}, \quad \forall w \in \operatorname{Supp} S_{i},\left\langle S_{i}, w\right\rangle=i .
$$

If $w$ is not in Supp $S_{i}$, set $\left\langle S_{i}, w\right\rangle=\mathbb{O}$ with the convention that $\mathbb{0}$ is neutral for both min and max and absorbing for + . We then consider the series $T$ defined 


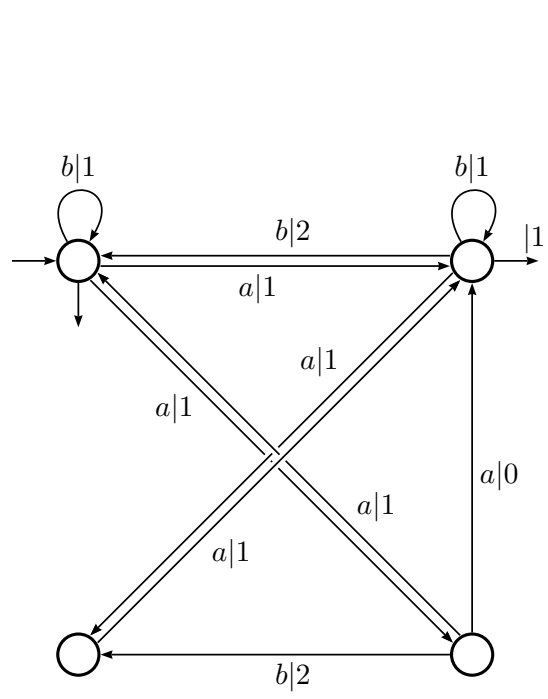

(a) The 1-valued automaton
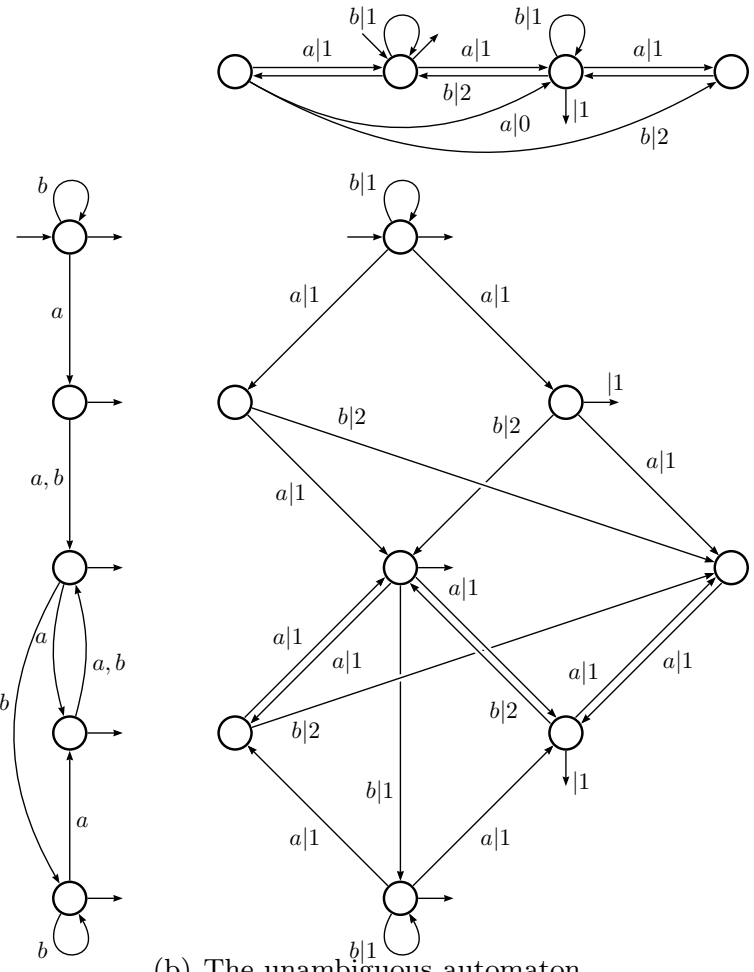

(b) The unambiguous automaton

Figure 2: Getting an unambiguous automaton (II)

by:

$$
\begin{aligned}
\forall w \in a^{*}, \quad & \left\langle T_{1}, w\right\rangle=\max \left(\left\langle S_{p}, w\right\rangle,\left\langle S_{q}, w\right\rangle\right), \quad\left\langle T_{2}, w\right\rangle=\min \left(\left\langle S_{r}, w\right\rangle,\left\langle S_{s}, w\right\rangle\right) \\
& \langle T, w\rangle=\left\langle T_{1}, w\right\rangle+\left\langle T_{2}, w\right\rangle .
\end{aligned}
$$

The series $T_{1}$ and $T_{2}$, and therefore $T$, are unambiguous, so they belong to $\mathbb{Z}_{\text {max }} \operatorname{Rat}\left(a^{*}\right) \cap \mathbb{Z}_{\text {min }} \operatorname{Rat}\left(a^{*}\right)$. The series $T_{1}$ is recognized by the max-plus au-

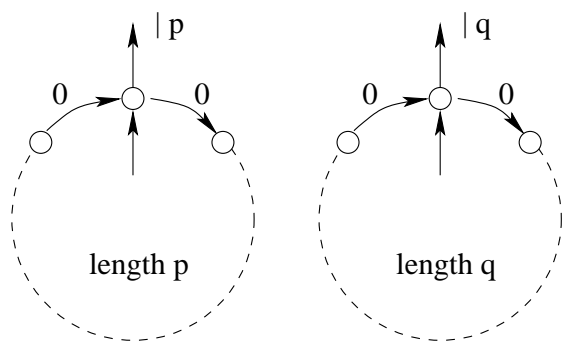

Figure 3: A max-plus automaton recognizing $T_{1}$.

tomaton of dimension $(p+q)$ given in Figure 3. A min-plus (and determinisic) automaton recognizing $T_{1}$ is the following one (for $p<q$ ): 
States: $\{0,1, \ldots, p q-1\}$; transitions: $i \stackrel{a \mid 0}{\longrightarrow} i+1 \bmod p q$; initial state: $\stackrel{\mid 0}{\rightarrow} 0$; final states: $i p \stackrel{\mid p}{\rightarrow}$ for $1 \leq i<q$, and $j q \stackrel{\mid q}{\rightarrow}$ for $0 \leq j<p$.

And similarly for $T_{2}$, the small automaton being the min-plus one. Therefore, the series $T$ is recognized by a max-plus automaton of dimension $(p+q) r s$, and a min-plus one of dimension $p q(r+s)$. Now observe that $\left(\left\langle T, a^{n}\right\rangle\right)_{n \in \mathbb{N}}$ is periodic of minimal period pqrs. Using the above claim, the smallest 1-valued (or unambiguous, or deterministic) automaton recognizing $T$ is of dimension pqrs.

\section{References}

[1] F. Baccelli, G. Cohen, G.J. Olsder, and J.P. Quadrat. Synchronization and Linearity. John Wiley \& Sons, New York, 1992.

[2] J. Berstel. Transductions and context-free languages. B. G. Teubner, 1979.

[3] A. Bonnier-Rigny and D. Krob. A complete system of identities for one-letter rational expressions with multiplicities in the tropical semiring. Theoretical Computer Science, 134:27-50, 1994.

[4] S. Eilenberg. Automata, languages and machines, volume A. Academic Press, New York, 1974.

[5] S. Gaubert and J. Mairesse. Modeling and analysis of timed Petri nets using heaps of pieces. IEEE Trans. Aut. Cont., 44(4):683-698, 1999.

[6] K. Hashigushi, K. Ishiguro, and S. Jimbo. Decidability of the equivalence problem for finitely ambiguous finance automata. Int. J. Algebra Comput., 12(3):445-461, 2002.

[7] J. Hopcroft and J. Ullman. Introduction to automata theory, languages, and computation. Addison-Wesley Publishing Co., 1979.

[8] I. Klimann, S. Lombardy, J. Mairesse, and C. Prieur. Deciding unambiguity and sequentiality from a finitely ambiguous max-plus automaton. Theoret. Comput. Sci., 2004. To appear. Short version in LNCS 2710:373-385, 2003.

[9] D. Krob. The equality problem for rational series with multiplicities in the tropical semiring is undecidable. Int. J. Algebra Comput., 4(3):405-425, 1994.

[10] D. Krob. Some consequences of a Fatou property of the tropical semiring. J. Pure Appl. Algebra, 93(3):231-249, 1994.

[11] M. Mohri. Finite-state transducers in language and speech processing. Comput. Linguist., 23(2):269-311, 1997. 
[12] $\mathrm{P}$. Moller. Théorie algébrique des systèmes à événements discrets. $\mathrm{PhD}$ thesis, École des Mines, Paris, 1988.

[13] J. Sakarovitch. A construction on finite automata that has remained hidden. Theoret. Comput. Sci., 204(1-2):205-231, 1998.

[14] M.-P. Schützenberger. Sur les relations rationnelles entre monoïdes libres. Theoret. Comput. Sci., 3(2):243-259, 1976/77.

[15] I. Simon. Recognizable sets with multiplicities in the tropical semiring. In Mathematical Foundations of Computer Science, Proc. 13th Symp., number 324 in LNCS, pages 107-120, 1988.

[16] L. Stockmeyer and A. Meyer. Word problems requiring exponential time: preliminary report. In Fifth Annual ACM Symposium on Theory of Computing, pages 1-9. Assoc. Comput. Mach., New York, 1973.

[17] A. Weber. Finite-valued distance automata. Theor. Comput. Sci., 134(1):225-251, 1994. 